\begin{tabular}{|c|c|}
\hline Title & A simple screening current simulation method using equival ent circuit model for REBCO pancake coils \\
\hline Author(s) & Noguchi, So; Imai, Teki; Park, Dongkeun; Hahn, Seungy ong; Iwasa, Y ukikazu \\
\hline Citation & $\begin{array}{l}\text { Superconductor science and technology, 33(11), } 115005 \\
\text { https://doi.org/10.1088/1361-6668/abb35b }\end{array}$ \\
\hline Issue Date & $2020-11$ \\
\hline DOC URL & http:/hdl.handle.net/2115/83145 \\
\hline Rights & $\begin{array}{l}\text { This is a peer-reviewed, un-copyedited version of an article accepted for publication/published in Superconductor } \\
\text { science and technology. IOP Publishing } \mathrm{L} \text { td is not responsible for any errors or omissions in this version of the } \\
\text { manuscript or any version derived from it. The Version of Record is available online at } 10.1088 / 1361-6668 / a b b 35 b .\end{array}$ \\
\hline Type & article (author version) \\
\hline File Information & SUST_SimpleSCIF_r1_081020-1.pdf \\
\hline
\end{tabular}

Instructions for use 


\title{
A Simple Screening Current Simulation Method Using Equivalent Circuit Model for REBCO Pancake Coils
}

\author{
So Noguchi' ${ }^{1}$, Teki Imai ${ }^{1}$, Dongkeun Park $^{2}$, Seungyong Hahn ${ }^{3}$, Yukikazu Iwasa ${ }^{2}$ \\ ${ }^{1}$ Graduate School of Information Science and Technology, Hokkaido University, Sapporo 060- \\ 0814, Japan. \\ ${ }^{2}$ Francis Bitter Magnet Laboratory, Plasma Fusion and Science Center, Massachusetts Institute \\ of Technology, Cambridge, MA 02139, USA. \\ ${ }^{3}$ Department of Electrical and Computer Engineering, Seoul National University, Seoul 08826, \\ South Korea. \\ E-mail: noguchi@ssi.ist.hokudai.ac.jp
}

\begin{abstract}
The screening current induced in rare-earth barium copper oxide (REBCO) tape generates an unwanted irregular magnetic field. The screening current-induced field (SCIF) is a challenging issue for MRI, NMR, and accelerators magnet composed of REBCO coils. A few FEM-based simulation methods have been proposed to estimate the SCIF; however, they require a long computation time.

Recently, we have proposed a simple SCIF computation method based on the self and mutual inductances of REBCO pancake coil and screening current radial paths on the top and bottom of pancake coils. Accuracy of the proposed method is not excellent; however, computation time is quite short. In this paper, we report an equivalent circuit model that includes the self and mutual inductances of a REBCO pancake coil and screening current radial path. Moreover, with this proposed method, we can compute SCIF of no-insulation (NI) REBCO pancake coils, while it is not the case with the previously proposed FEM-based simulation method. The proposed method has been validated by experiment. The proposed method is available online.
\end{abstract}

\section{Introduction}

Recent years, the second generation high-temperature superconducting $\left[(\mathrm{RE}) \mathrm{Ba}_{2} \mathrm{Cu}_{3} \mathrm{O}_{y}(\mathrm{RE}=\mathrm{Rare}\right.$ Earth, REBCO)] coated conductors are used for some high-field magnet applications, such as magnetic resonance imaging (MRI) [1-3], nuclear magnetic resonance (NMR) [4, 5], and particle accelerators [6, 7]. Although such applications require an accurate magnetic field, a screening current induced in REBCO tape degrade field quality [8-10]. A few simulation methods based on finite element method (FEM) coupling with thin approximation method $[11,12]$ have been developed in order to estimate the screening current-induced field (SCIF). However, the FEM-based simulation method is complicated and difficult to be applied. Unless a special technique, such as the fast multipole method [13-15], is employed, an enormous size of PC memory and an inordinately long computation time are required.

To shorten a computation time and reduce the required PC memory without special technique, we have developed a simple SCIF computation method [16] that can easily give SCIF from the coil configurations. Although our crude model does not result SCIF as accurate as those by FEM-based methods. It takes a very short time to simulate an SCIF, and the method is very simple.

In this paper, we have improved the previously proposed method to simulate SCIF under various conditions, including for no-insulation (NI) REBCO pancake coils [17]. The current FEM-based SCIF simulation method cannot be applied to NI REBCO pancake coils: to make it applicable the 
circumferential current must be known. Despite the screening current is computed from the circumferential current, the currents in the circumferential and radial directions are not simulated in the current FEM-based SCIF simulation method. In our method, to simultaneously compute the circumferential and radial currents and the screening current, the screening current inductance derived in [16] is directly coupled to the equivalent electric circuits of NI REBCO pancake coil $[17,18]$. The time-varying SCIF can be obtained even while NI REBCO pancake coils are being charged. To confirm the validity of our proposed method, we compared three SCIF results, those computed by our method, FEM, and measurement. Our SCIF simulation tool is provided online.

\section{Screening Current-Induced Magnetic Field Simulation Method}

Figure 1 shows the proposed equivalent electric circuit of the $i$ th (top) and the $j$ th (bottom) pancake coils that includes an equivalent screening current circuit. Here, the screening current inductance is given by [16]

$$
L_{\mathrm{sc}}=\frac{\mu_{0} N^{2} \hat{S}}{d}
$$

where $\mu_{0}$ and $N$ are the magnetic permeability of free space, the number of turns. As indicated in figure $2, \hat{S}$ is the average $r$-directed REBCO tape surface area per turn and $d$ is REBCO single pancake coil thickness; $\hat{S}$ is given by:

$$
\hat{S}=2 \pi \hat{r} w
$$

where $\hat{r}$ and $w$ are the average coil radius and the tape width, respectively.

Next, the mutual inductance between the screening currents $I_{\mathrm{sc} i}$ and $I_{\mathrm{sc} j}$ is

$$
M_{\mathrm{sci}, \mathrm{sc} j}=-\frac{N_{i} \Phi_{\mathrm{sci}, \mathrm{sc} j}}{I_{\mathrm{sc} j}}=-\hat{b}_{\mathrm{sci} i, \mathrm{sc} j} \hat{S}_{i} N_{i}
$$

where $\Phi_{\text {sci,sc } j}$ and $\hat{b}_{\text {sci,sc } j}$ are the magnetic flux penetrating to the REBCO tape surface of pancake coil $i$ generated from screening current $I_{\mathrm{sc} j}$ and the average radial field on pancake coil $i$ generated by $I_{\mathrm{sc} j}=$ $1 \mathrm{~A}$, respectively. With the screening current assumed to flow only along the REBCO tape edges as shown in figure $3, \hat{b}_{\text {scisc } j}$ is obtained as follows:

$$
\hat{b}_{\text {sci,sc } j}=\frac{\mu_{0}}{4 \pi} \int_{r_{0 j}}^{r_{1 j}} \int_{0}^{2 \pi} J_{\text {sc } j}\left\{\frac{\hat{z}_{i}-z_{1 j}}{\left|\boldsymbol{D}\left(z_{1 j}\right)\right|^{3}}-\frac{\hat{z}_{i}-z_{0 j}}{\left|\boldsymbol{D}\left(z_{0 j}\right)\right|^{3}}\right\} r \mathrm{~d} \theta \mathrm{d} r
$$

Referring all parameters in equation 4 to the $i$ th pancake coil, we have: $r_{0 j}$ and $r_{1 j}$, the inner and outer radii; $z_{0 j}$ and $z_{1 j}$, the bottom and top $z$-positions; and $\hat{z}_{i}$ the average $z$-position. We have:

$$
\begin{gathered}
J_{\mathrm{sc} j}=\frac{N_{j}}{r_{1 j}-r_{0 j}} \\
\boldsymbol{D}(z)=\left(\hat{r}_{i}-r \cos \theta,-r \sin \theta, \hat{z}_{i}-z\right) .
\end{gathered}
$$

Finally we compute $\hat{b}_{\text {sci,scj }}$ using numerical integration technique.

Similarly, the mutual inductance between the screening currents $I_{\mathrm{sc} i}$ and the coil current $I_{\theta j}$ (as shown in figure 4) is represented by

$$
M_{\mathrm{sci}, \theta j}=-\frac{N_{i} \Phi_{\mathrm{sci} i \theta j}}{I_{\theta j}}=-\hat{b}_{\mathrm{sc} i, \theta j} \hat{S}_{i} N_{i}
$$

where $\Phi_{s c i, \theta j}$ is the magnetic flux penetrating to the REBCO tape surface of $i$ th pancake coil generated from the pancake coil $I_{\theta j}$, and

$$
\begin{gathered}
\hat{b}_{\mathrm{sci}, \theta j}=\frac{\mu_{0}}{4 \pi} \int_{z_{0 j}}^{z_{1 j}} \int_{r_{0 j}}^{r_{1 j}} \int_{0}^{2 \pi} J_{\theta j} \frac{\hat{z}_{i}-z}{|\boldsymbol{D}(z)|^{3}} r \mathrm{~d} \theta \mathrm{d} r \mathrm{~d} z \\
J_{\theta j}=\frac{N_{j}}{w\left(r_{1 j}-r_{0 j}\right)} .
\end{gathered}
$$

Finally, to decide the resistance on the screening current circuit $R_{\mathrm{sc}}$, we suppose the followings in this analysis:

- The screening current direction are opposite on the both REBCO tape edges. 
- The resistance on the screening current flowing in the same direction as that of transport current is $R_{\mathrm{sc}}$;

- The resistance on the screening current in the opposite direction as that of the transport current is ignored.

Therefore, considering the right half tape in figure 5 , a current of $0.5 I_{\theta}+I_{\mathrm{sc}}$ flows there. Supposing that the resistance of left side is zero, $R_{\mathrm{sc}}$ is twice longitudinal tape resistance:

$$
R_{\mathrm{sc}}=2 R_{\mathrm{su}}
$$

where, according to the $n$-power law, $R_{\text {su }}$ is obtained from

$$
R_{\mathrm{su}}=\ell \frac{E_{\mathrm{c}}}{I_{\mathrm{c}}}\left(\frac{I_{\theta}+2 I_{\mathrm{sc}}}{I_{\mathrm{c}}}\right)^{n-1}
$$

where $\ell, I_{\mathrm{c}}$, and $E_{\mathrm{c}}$ are the pancake coil REBCO tape length of pancake coil, the critical current, and the electric field at the critical current, respectively.

The other parameters, $L, R_{\mathrm{mt}}$, and $R_{\mathrm{sc}}$ in figure 1, are obtained from the magnet shape, the tape configuration [19] or experiments.

\section{SCIF Simulation results}

To investigate the validity of the proposed SCIF simulation method, the SCIF results are compared, FEM-based simulation vs. experiment. Three magnets are tested: 1) composed of two double-pancake and four single pancake coils [16, 20]; 2) LBC3 insert magnet; and 3) Coil 2 of a 3-coil HTS insert of the MIT 1.3-GHz NMR magnet.

\subsection{Two double-pancake and four single pancake coils (All turns insulated)}

We compared the simulation and measurement results of the magnet composed of two double-pancake and four single pancake $(2 \mathrm{D}+4 \mathrm{~S})$ coils $[16,20]$ with those of a FEM simulation, which is a $2 \mathrm{D}$ finite element method with thin approximation method (FEM+TAM) [11, 12]. Table 2 and figure 6(a) show the magnet configuration, in which turns of all pancake coils are insulated between turns. In our experiments, we energized the magnet to $30 \mathrm{~A}$ in $10 \mathrm{~min}$., with its coils charged in 3 ways, as shown in figure 6(b)-(d): Case I [figure 6(b)], all coils (DPs $1 \& 2+$ SPs 1-4); Case II [figure 6(c)], DPs $1 \& 2$ and SPs $1 \& 2$; Case III [figure 6(d)] DPs $3 \& 4$.

Figure 7 shows the simulation and measurement, and FEM+TAM results. Here, the SCIF $B_{\mathrm{sc}}$ is defined as follows:

$$
B_{\mathrm{sc}}=B_{0}^{\mathrm{exp}}-B_{0}^{\mathrm{sim}}
$$

where $B_{0}^{\text {exp }}$ and $B_{0}^{\text {sim }}$ are both center magnetic fields: $B_{0}^{\text {exp }}$, measured; $B_{0}^{\text {sim }}$, computed without screening current contribution. In each case, simulation results of our proposed method and FEM+TAM agree well, but they differ from measurement, particularly for Case II. Due to a small SCIF signal level, we could not measure SCIF accurate enough to definitely compare simulation and measurement results. Good agreement between our proposed method and FEM+TAM indicates despite its simple computation, thus a significantly shorter computation time, our proposed method is as accurate as FEM+TAM method.

\subsection{LBC3 magnet (No-insulation coil)}

The LBC3 magnet is the insert of a magnet that generated a center magnetic field of $45.5 \mathrm{~T}$ at National High Magnetic Field Lab, USA [21]. Figure 8 shows a schematic drawing of the LBC3 magnet that consists of 12 no-insulation REBCO single pancake coils; table 3 lists its specifications.

Table 4 lists the SCIFs computed by the proposed method and the FEM+TAM simulation together with the measurements. The results of the proposed method do not agree with the measurement well. However, the accuracy is almost the same as the FEM+TAM simulation. Although the accuracy of the proposed method is not so high due to rough modelling, it is possible to know the level of the SCIF. The validity of the proposed method is confirmed.

Figure 9 gives the magnetic field graph, which is the screen copy of online service "SCIFweb service" as described in Appendix, when the test coil charges up to $50 \mathrm{~A}$ with $500 \mathrm{~mA} / \mathrm{s}$ at $4.2 \mathrm{~K}$. In figure $9, I_{\mathrm{op}}$, 
$B_{\text {ideal }}, B_{\text {sc }}$, and $B_{\text {total }}$ mean the transport current, the magnetic field without screening current, the screening current-induced field, and the magnetic field with screening current, respectively. The LBC3 magnet has no insulation between turns, and it is supposed that the turn-to-turn contact resistivity is 7 $\mu \Omega \cdot \mathrm{cm}^{2}$ in simulations. Not only the difference between the ideal and real magnetic field but the charging delay of magnetic field are observable. Since the FEM+TAM simulation method cannot take into account the contact resistance of NI REBCO pancake coils, only the proposed method can deal with NI REBCO pancake coils so far.

\subsection{Coil 2 of 3-nested 800-MHz REBCO insert of the MIT 1.3-GHz NMR magnet (No-insulation coil)}

The Francis Bitter Magnet Laboratory (FBML) of MIT built and tested the 800-MHz NI REBCO insert (H800) for the MIT 1.3-GHz LTS/HTS NMR magnet [4] H800 was unexpectedly quenched and damaged during operation, and the new designed REBCO insert, in which unanticipated flaws of $\mathrm{H} 800$ are eliminated, is under development [22, 23]. H800 consists of 3 nested coils, Coils 1, 2, and 3. Here, the SCIF of Coil 2 was simulated and compared with the measurement [24]. The specifications of Coil 2 are shown in table 5 .

Figure 10 shows the magnetic field transition energized up to $251.3 \mathrm{~A}$ with $2 \mathrm{~A} / \mathrm{min}$. The measured center field was approximately $5.58 \mathrm{~T}$ at a temperature of $4.2 \mathrm{~K}$. The simulated fields with/without the screening current are 5.594 $\mathrm{T}$ and 5.635 T, respectively. The field with screening current is closer to the measurements. Figure 11 represents the simulated field distribution on $z$-axis, and it well agrees with the measured distribution shown in [24]. From the comparison between the simulation results and the measurement, the validity of the proposed simulation method is confirmed.

\section{Conclusion}

In this paper, we present a simple screening current-induced field (SCIF) computation method by using a circuit model composed of the circuit elements of pancake coils and screening current radial paths. Having derived coil inductances required in the proposed method [19], we derive in this paper the inductance and resistance of the screening current radial paths. Although our assumption of screening current paths is crude, our simulation results are sufficiently accurate, and equally important, its computation time is notably shorter than the FEM-based computation [11, 12].

To check the accuracy of our proposed method, the SCIF of three different magnets, 4D+2S, LBC3, and Coil 2 of a 3 -coil $800-\mathrm{MHz}$ insert of a $1.3-\mathrm{GHz}$ LTS/HTS magnet, were computed and compared with the measurement and the FEM-based simulation results. The proposed method has a sufficient accuracy, but not accurate enough as a design tool for MRI/NMR magnet. Because SCIF results by measurement and simulation agree well enough, and requires short computation time, our proposed method is suitable for first-cut design phase. For higher accuracy, we need to use more realistic REBCO tape properties and subdivide pancake coils in the radial direction as was done in a moderate model [19].

The proposed SCIF computation system is posted online. In the near future, we will build on this model by adding temperature and stress functions.

\section{Acknowledgement}

This work was supported in part by the JSPS KAKENHI under Grant 20H02125.

D. Park and Y. Iwasa in this work were supported by the National Institute of General Medical Sciences of the National Institutes of Health under award number R01GM137138-01.

\footnotetext{
Appendix

The developed system is provided on a web site, named "SCIFweb service", at https://hbd.ist.hokudai.ac.jp/scifweb/. In the simulation system, the specifications of REBCO tape are referred by [25]. Since the paper [25] gives the specifications from 30 to $77 \mathrm{~K}$, the critical current is extrapolated below $30 \mathrm{~K}$. The system produces the simulation data as CSV data.
}

\section{Reference}


[1] Tosaka T, Miyazaki H, Iwai S, Otani Y, Takahashi M, Tasaki K, Nomura S, Kurusu T, Ueda H, Noguchi S, Ishiyama A, Urayama S and Fukuyama H 2016 R\&D project on HTS magnets for ultrahigh-field MRI systems IEEE Trans. Appl. Supercond. 264402505

[2] Miyazaki H, Iwai S, Otani Y, Takahashi M, Tosaka T, Tasaki K, Nomura S, Kurusu T, Ueda H, Noguchi S, Ishiyama A, Urayama S and Fukuyama H 2016 Design of a conduction-cooled 9.4T REBCO magnet for whole-body MRI systems Supercond. Sci. Technol. 29104001

[3] Yokoyama S, Lee J, Imura T, Matsuda T, Eguchi R, Inoue T, Nagahiro T, Tanabe H, Sato S, Daikoku A, Nakamura T, Shirai Y, Miyagi D and Tsuda M 2017 Research and Development of the High Stable Magnetic Field ReBCO Coil System Fundamental Technology for MRI IEEE Trans. Appl. Supercond. 274400604

[4] Iwasa Y, Bascuñán J, Hahn S, Voccio J, Kim Y, Lécrevisse T, Song J and Kajikawa K 2015 A high-resolution 1.3-GHz/54-mm LTS/HTS NMR magnet IEEE Trans. Appl. Supercond. 25 4300205

[5] Iguchi S, Piao R, Hamada M, Matsumoto S, Suematu H, Takao T, Saito A T, Li J, Nakagome H, Jin X, Takahiashi M, Maeda H and Yanagisawa Y 2016 Advanced field shimming technology to reduce the influence of a screening current in a REBCO coil for a high-resolution NMR magnet Supercond. Sci. Technol. 29045013

[6] Ueda H, Fukuda M, Hatanaka K, Wang T, Wang X, Ishiyama A, Noguchi S, Nagaya S, Kashima $\mathrm{N}$ and Miyahara N 2013 Conceptual design of next generation HTS cyclotron IEEE Trans. Appl. Supercond. 234100205

[7] Nugteren J, Kirby G, Murtomäki J, DeRijk G, Rossi L and Stenvall A 2018 Toward REBCO 20 $\mathrm{T}+$ dipoles for accelerators IEEE Trans. Appl. Supercond. 284008509

[8] Miyazaki H, Iwai S, Uto T, Otanii Y, Takahashi M, Tosaka T, Tasaki K, Nomura S, Kurusu T, Ueda H, Noguchi S, Ishiyama A, Urayama S and Fukuyama H 2017 Screening-current-induced magnetic field of conduction-cooled HTS magnets wound with REBCO-coated conductors IEEE Trans. Appl. Supercond. 274701705

[9] Uglietti D, Yanagisawa Y, Maeda H and Kiyoshi T 2010 Measurements of magnetic field induced by screening currents in YBCO solenoid coils Supercond. Sci. Technol. 23115002

[10] Dilasser G, Fazilleau P and Tixador P 2017 Experimental measurement and numerical simulation of the screening current-induced field decay in a small ReBCO coil IEEE Trans. Appl. Supercond. 274900104

[11] Ueda H, Fukuda M, Hatanaka K, Wang T, Ishiyama A and Noguchi S 2013 Spatial and temporal behavior of magnetic field distribution due to shielding current in HTS coil for cyclotron application IEEE Trans. Appl. Supercond. 234100805

[12] Noguchi S, Hahn S, Ueda H, Kim S and Ishiyama A 2018 An extended thin approximation method to simulate screening current induced in REBCO coils IEEE Trans. Magn. 547201904

[13] Rokhlin V 1983 Rapid solution of integral equations of classical potential theory J. Comput. Phys. $60187-21$

[14] Greengard L and Rokhlin V 1997 A new version of fast multipole method for the Laplace equation in three dimensions Acta Numerica 6 229-41

[15] Takahashi Y and Wakao S 2006 Large-scale analysis of eddy-current problems by the hybrid finite element-boundary element method combined with the fast multipole method IEEE Trans. Magn. 42 671-4

[16] Noguchi S, Ueda H, Hahn S, Ishiyama A and Iwasa Y 2019 A simple screening current-induced magnetic field estimation method for REBCO pancake coils Supercond. Sci. Technol. 32045007

[17] Hahn S, Park D K, Bascuñán J and Iwasa Y 2011 HTS pancake coils without turn-to-turn insulation IEEE Trans. Appl. Supercond. 21 1592-4

[18] Wang X, Hahn S, Kim Y, Bascuñán J, Voccio J, Lee H and Iwasa Y 2013 Turn-to-turn contact characteristics for an equivalent circuit model of no-insulation ReBCO pancake coil Supercond. Sci. Technol. 26035012 
[19] Noguchi S 2019 Electromagnetic, Thermal, and Mechanical Quench Simulation of NI REBCO Pancake Coils for High Magnetic Field Generation IEEE Trans. Appl. Supercond. 294602607

[20] Mochida A, Ueda H, Noguchi S, Wang T, Ishiyama A, Miyazaki H, Tosaka T, Nomura S, Kurusu T, Urayama S and Fukuyama H 2016 Evaluation of magnetic field distribution by screening current in multiple REBCO coils IEEE Trans. Appl. Supercond. 264702805

[21] Hahn S, Kim K, Kim K, Hu X, Painter T, Dixon I, Kim S, Bhattarai K, Noguchi S, Jaroszynski J and Larbalestier D 2019 45.5-tesla direct-current magnetic field generated with a hightemperature superconducting magnet Nature 560 496-4

[22] Park D, Bascuñán J, Michael P C, Lee J, Hahn S and Iwasa Y 2018 Construction and test results of coils 2 and 3 of a 3-nested-coil 800- MHz REBCO insert for the MIT 1.3-GHz LTS/HTS NMR magnet IEEE Trans. Appl. Supercond. 284300205

[23] Michael P C, Park D, Choi Y H, Lee J, Li Y, Bascuñán J, Noguchi S, Hahn S and Iwasa Y, 2019 Assenbly and test of 3-nested-coil 800-MHz REBCO insert (H800) for the MIT $1.3 \mathrm{GHz}$ LTS/HTS NMR magnet IEEE Trans. Appl. Supercond. 294300706

[24] Park D, Bascuñán J, Michael P C, Lee J, Choi Y H, Li Y, Hahn S and Iwasa Y 2019 MIT 1.3GHz LTS/HTS NMR magnet: Post quench analysis and new 800-MHz insert design IEEE Trans. Appl. Supercond. 294300804

[25] Ueda H, Imaichi Y, Wang T, Ishiyama A, Noguchi S, Iwai S, Miyazaki H, Tosaka T, Nomura S, Kurusu T, Urayama S and FukuyamaH 2014 Measurement and simulation of magnetic field generated by screening currents in HTS coil IEEE Trans. Appl. Supercond. 244701505 

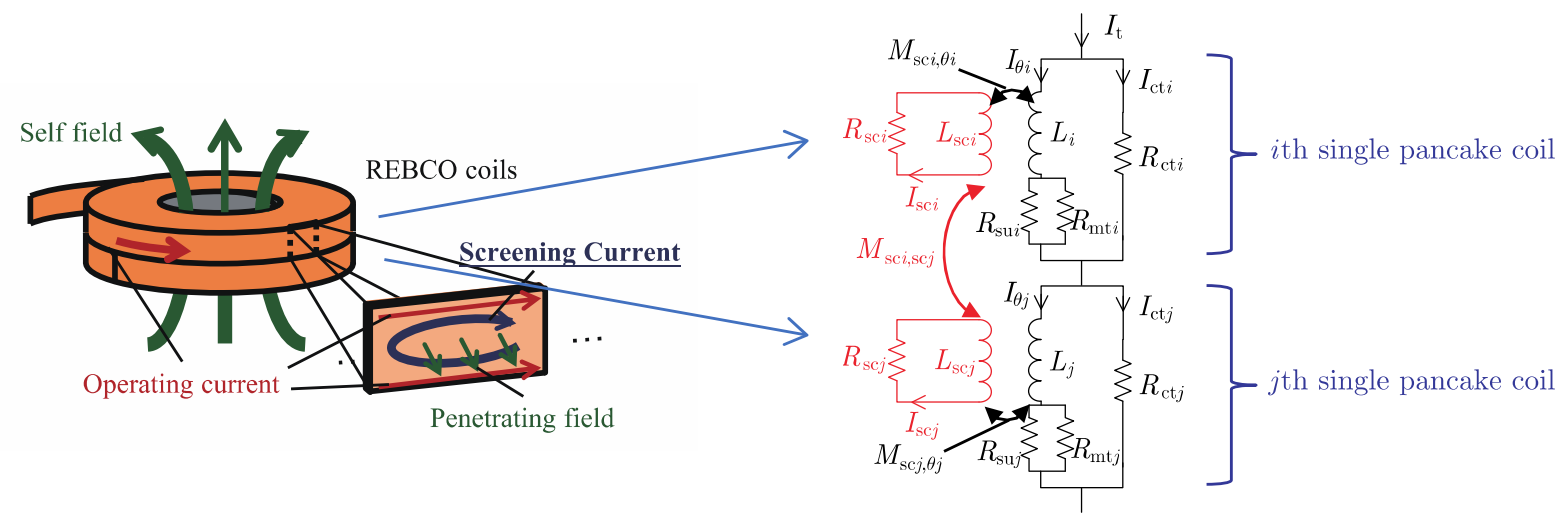

Figure 1. Equivalent electric circuit of the $i$ th (top) and the $j$ th (bottom) pancake that includes an equivalent screening current circuits. The symbols are listed in table 1.

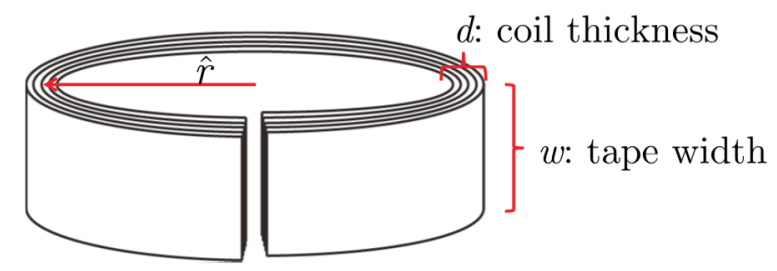

Development view

$$
2 \pi \hat{r}
$$

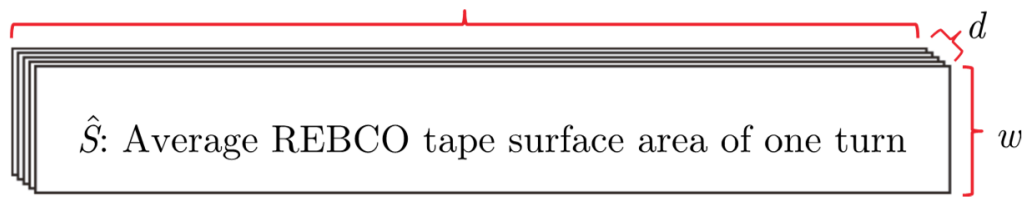

Figure 2. Pancake coil parameters necessary for derivation of screening current inductance.

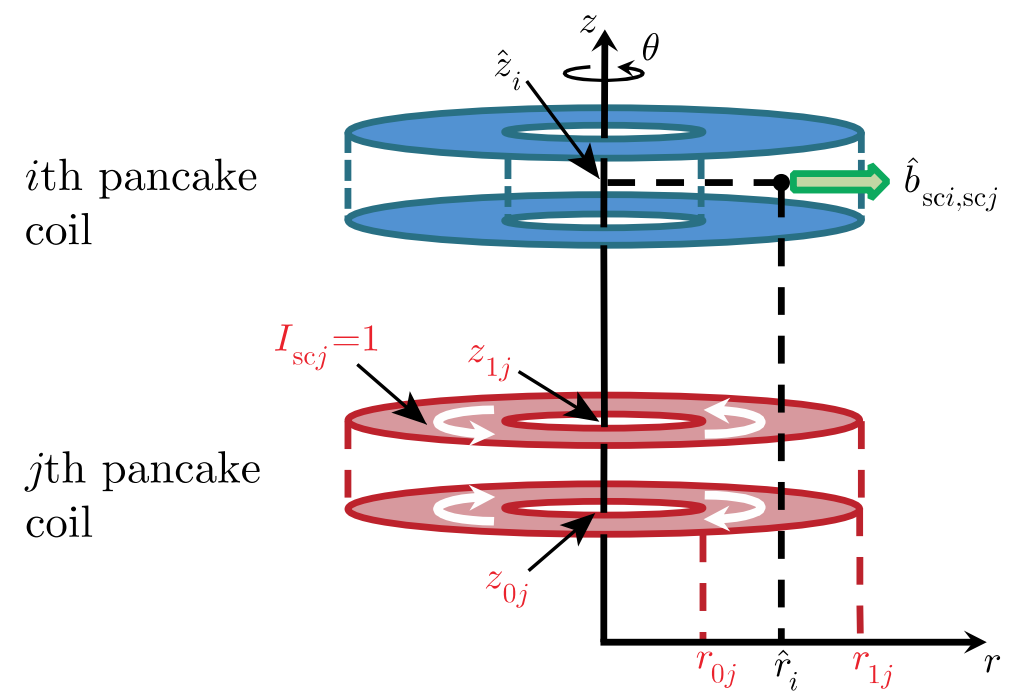

Figure 3. Pancake coil parameters necessary for derivation of screening current inductance. 


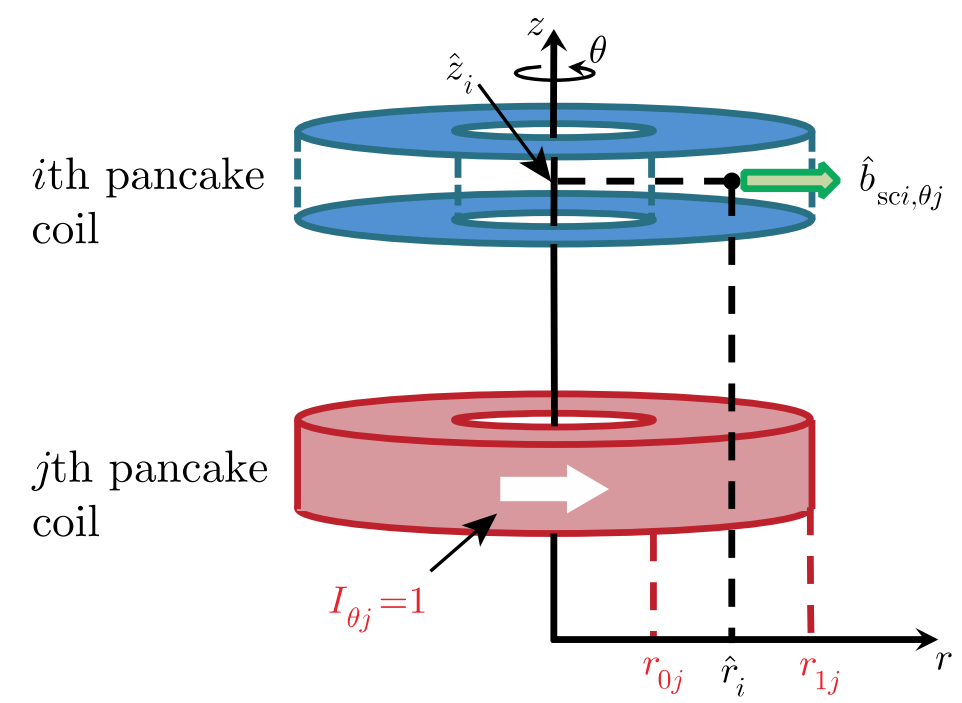

Figure 4. Pancake coil parameters necessary for derivation of screening current inductance and pancake coil.

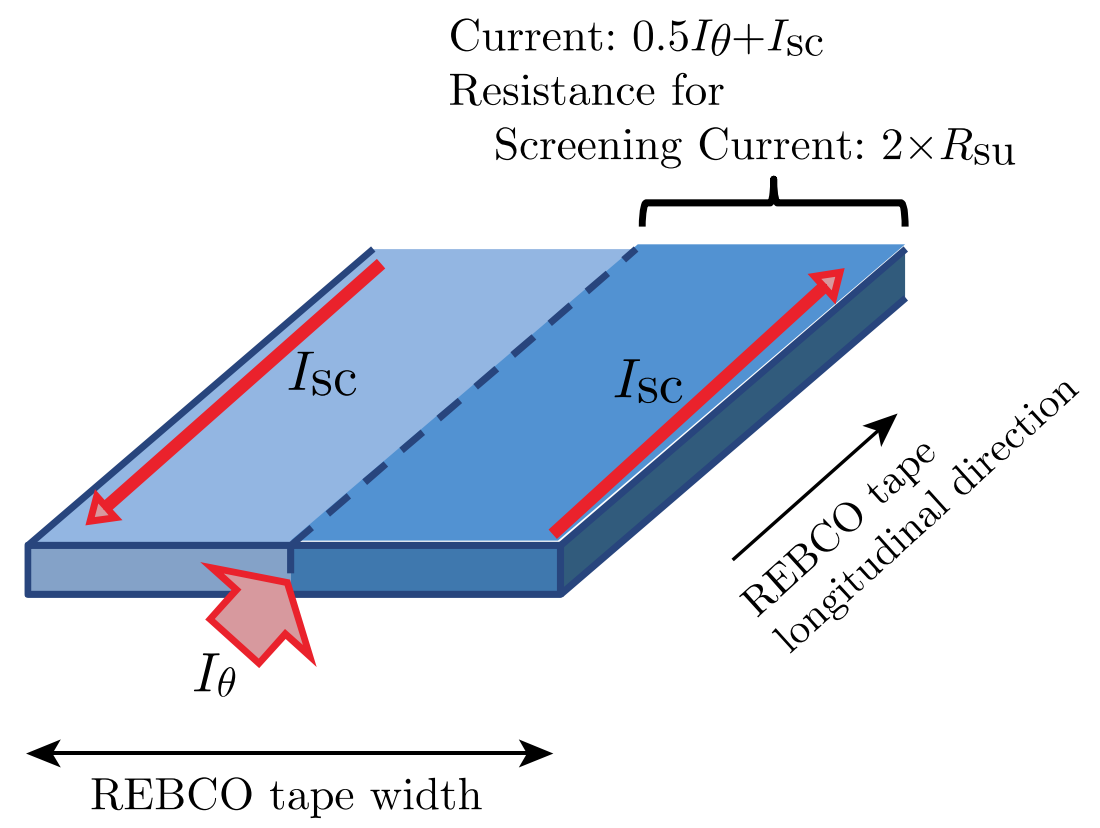

Figure 5. Current flowing illustration, considering operating current and screening current. On right half side, a current of $0.5 I_{\theta}+I_{\mathrm{sc}}$ carries. 


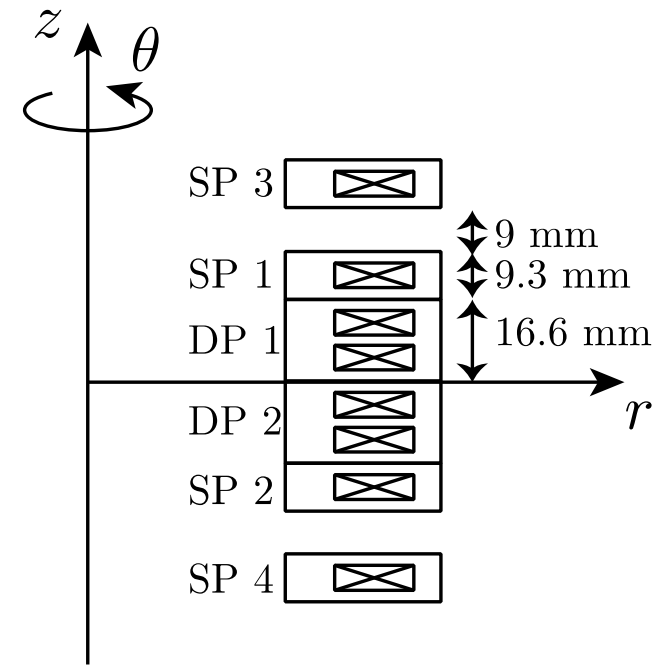

(a)

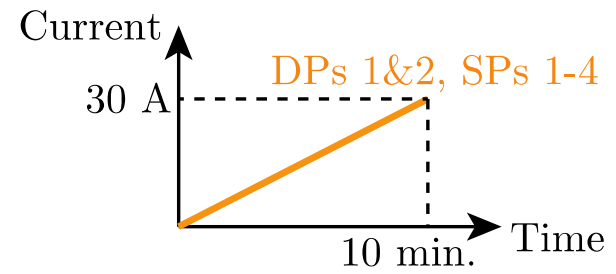

(b) Case I

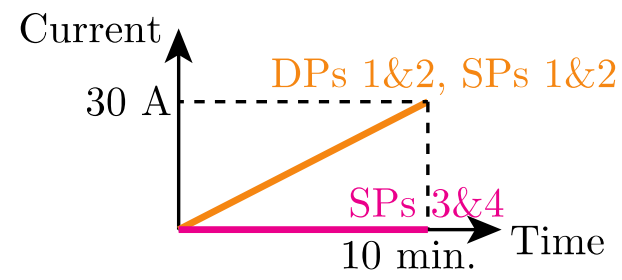

(c) Case II

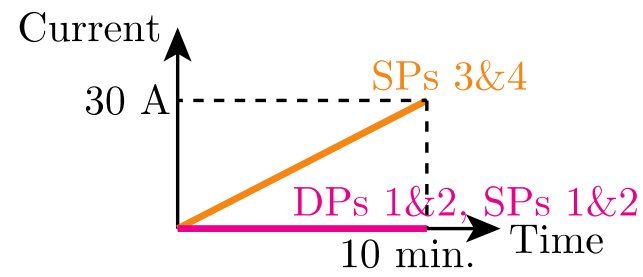

(d) Case III

Figure 6. (a) Cross-sectional view of 2 double and 4 single pancake (2DP+4SP) coils. (b)-(d) Three charging patterns for measuring the SCIF. 


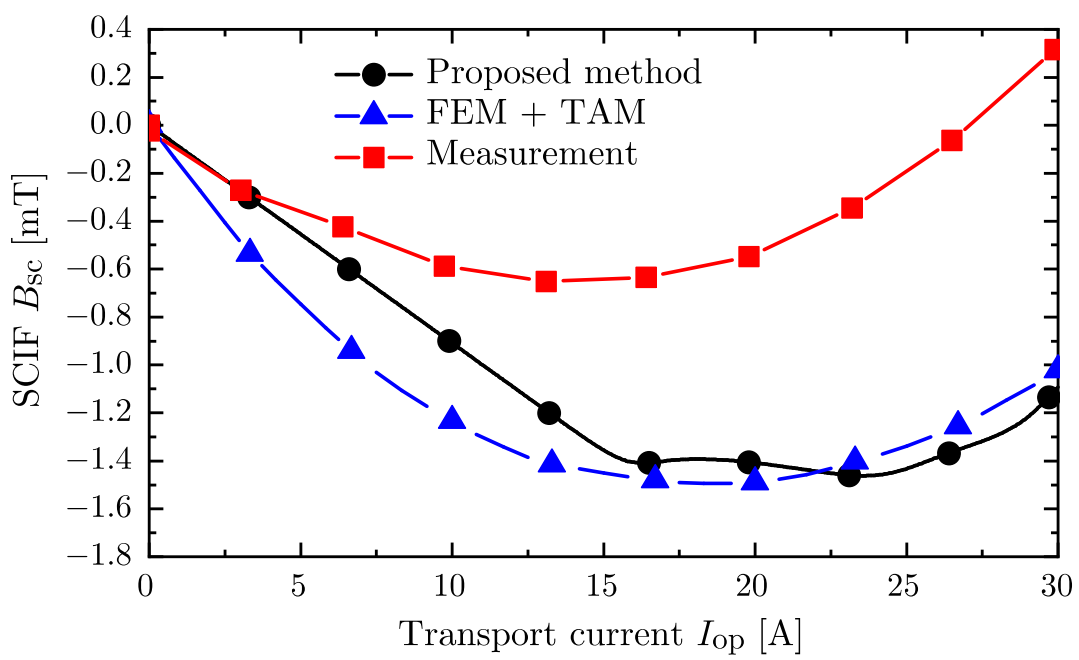

(a) Case I

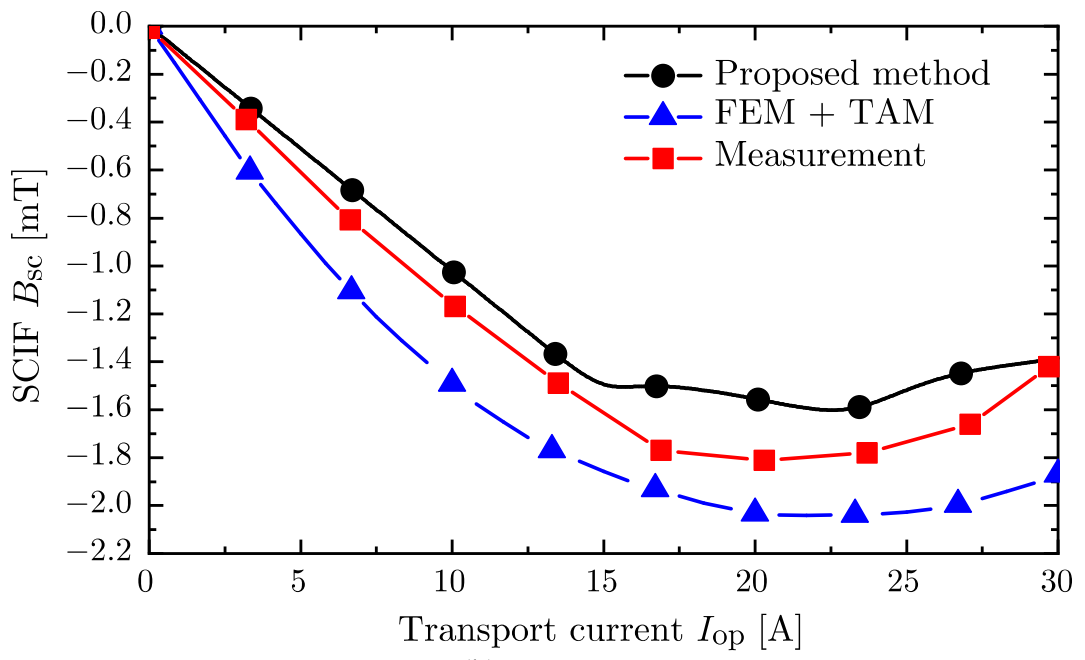

(b) Case II

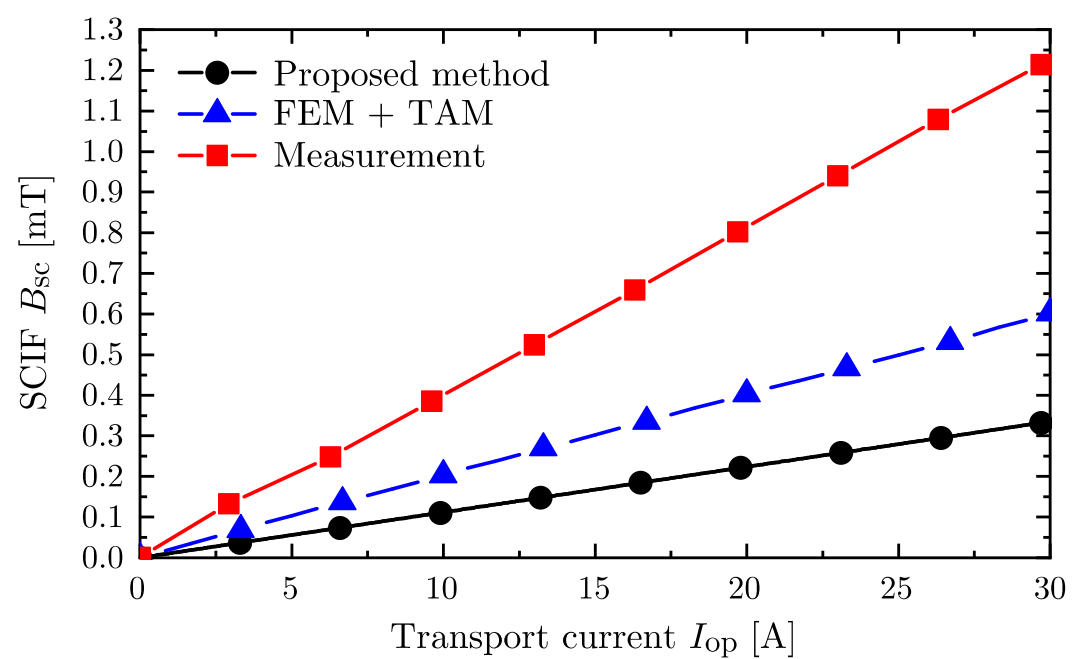

(c) Case III

Figure 7. SCIFs at magnet center of $2 \mathrm{D}+4 \mathrm{SP}$ coil by the proposed method, FEM+TAM, and measurement on three different energizing patterns; (a) case I, (b) case II, and (c) case III, respectively. 


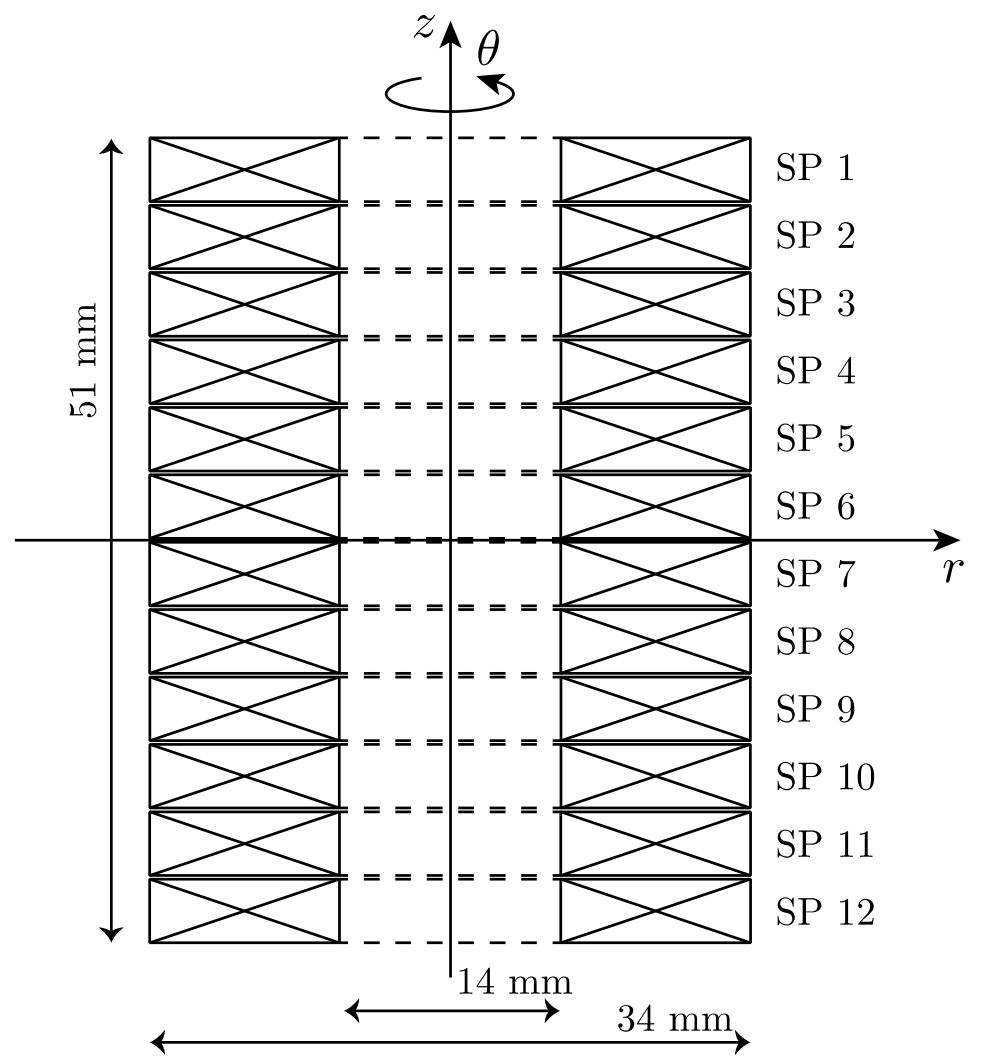

Figure 8. Schematic drawing of the LBC3 magnet consisting of 12 REBCO single pancake coils.

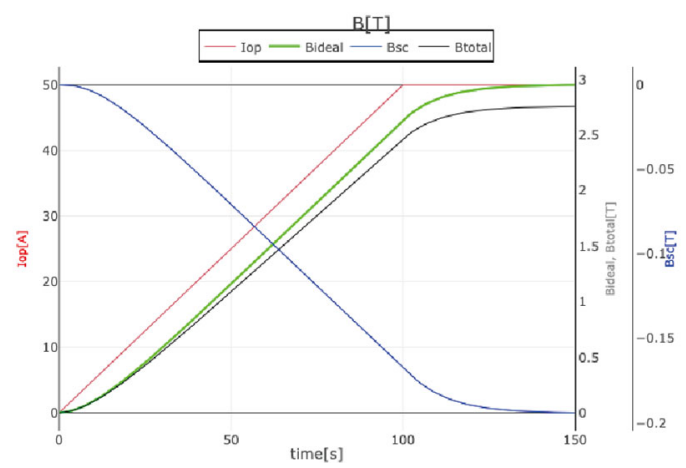

Figure 9. Center magnetic field with and without screening current, $B_{\text {total }}$ and $B_{\text {ideal, }}, \mathrm{SCIF} B_{\mathrm{sc}}$, and transport current $I_{\mathrm{op}}$ of LBC3 insert magnet (captured from the online site shown in Appendix). 


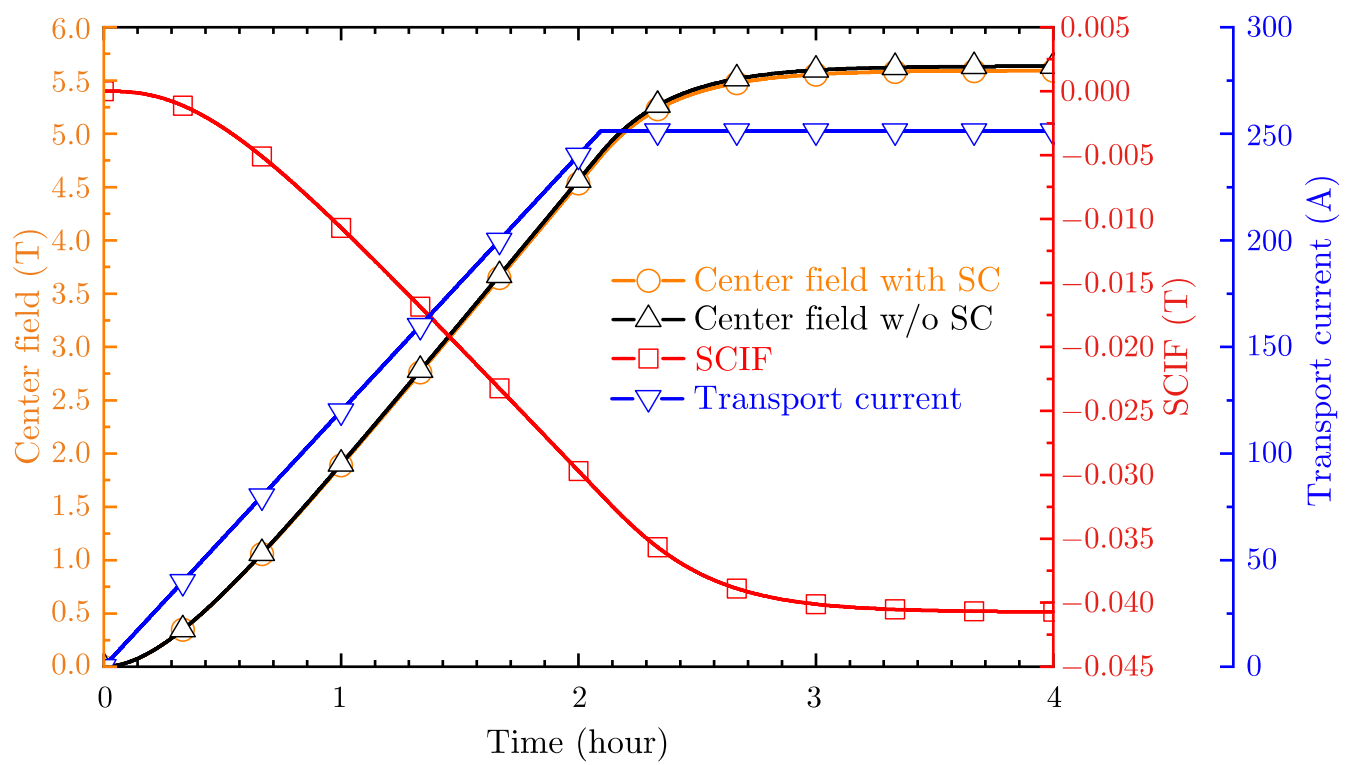

Figure 10. Simulated center field with/without screening current (SC), SCIF, and transport current of Coil 2 of $800-\mathrm{MHz}$ REBCO insert magnet.

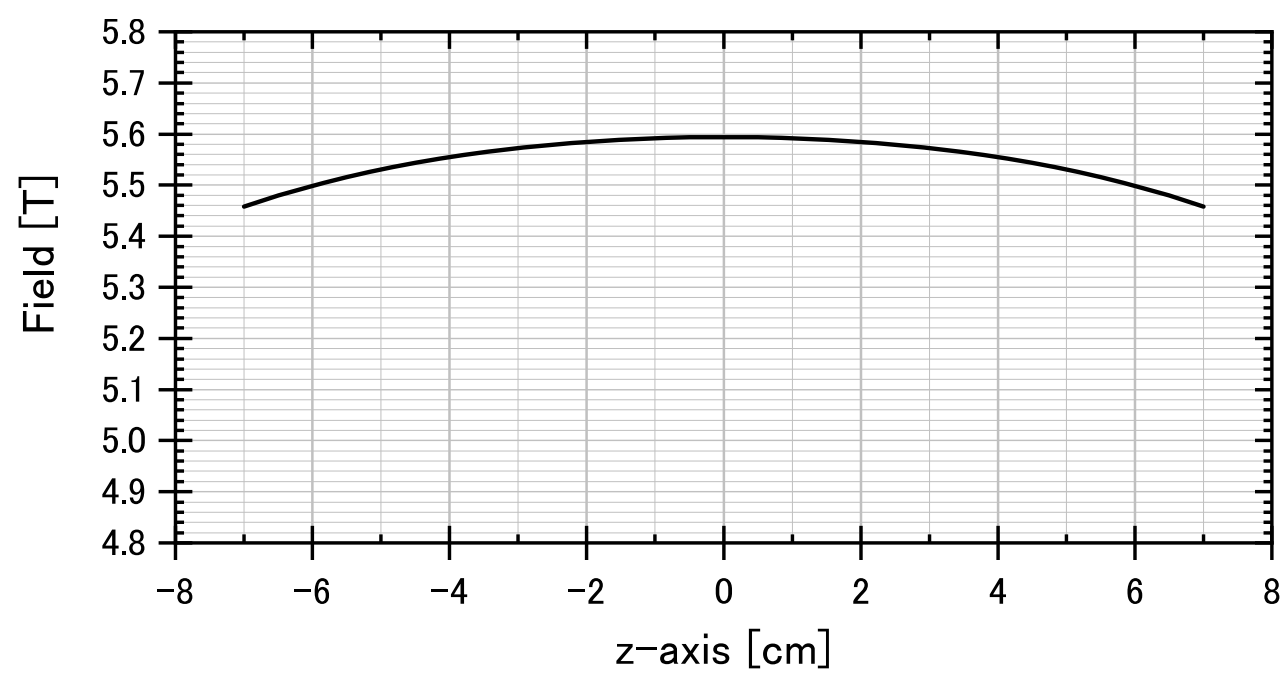

Figure 11. Profile of Coil 2 axial field distribution. 
Table 1. Symbols in equivalent circuit

\begin{tabular}{cl}
\hline$I_{\mathrm{t}}$ & Transport current \\
$I_{\theta}$ & Azimuthal coil current \\
$I_{\mathrm{ct}}$ & Radial current in case of NI coil \\
$L$ & Coil self-inductance \\
$L_{\mathrm{sc}}$ & Screening current self-inductance \\
$M_{\mathrm{sc}, \theta}$ & Mutual inductance between azimuthal coil current \\
& and screening current \\
$M_{\mathrm{sc}, \mathrm{sc}}$ & Mutual inductance between screening currents \\
$R_{\mathrm{su}}$ & Equivalent resistance of superconducting layer \\
$R_{\mathrm{mt}}$ & Resistance of matrix metal \\
$R_{\mathrm{sc}}$ & Resistance for screening current \\
$R_{\mathrm{ct}}$ & Turn-to-turn contact resistance in case of NI \\
\hline
\end{tabular}

Table 2. 2 DP and 4 SP coil specifications

\begin{tabular}{lcccccc}
\hline & DP 1 & DP 2 & SP 1 & SP 2 & SP 3 & SP 4 \\
\hline REBCO tape width [mm] & 4.0 & 4.0 & 4.0 & 4.0 & 4.0 & 4.0 \\
REBCO tape thickness [mm] & 0.1 & 0.1 & 0.1 & 0.1 & 0.1 & 0.1 \\
Inner radius [mm] & 50.0 & 50.0 & 50.0 & 50.0 & 50.0 & 50.0 \\
Outer radius [mm] & 63.8 & 63.6 & 62.8 & 63.4 & 63.0 & 62.9 \\
Coil height [mm] & 16.5 & 16.5 & 9.3 & 9.3 & 9.3 & 9.3 \\
Number of turns [turn] & 110 & 110 & 111 & 111 & 111 & 110 \\
REBCO tape $I_{\mathrm{c}}[\mathrm{A}$ ] at 77 K, s.f. & 118.0 & 119.9 & 91.0 & 64.0 & 65.0 & 65.1 \\
$n$-value & 26.05 & 28.80 & 23.46 & 23.11 & 22.04 & 25.43 \\
Turn-to-turn insulation & \multicolumn{7}{c}{ Co-winding with Kapton® 25 $\mu \mathrm{m}$} \\
Cooling condition & \multicolumn{7}{c}{ Immersion in liquid nitrogen } \\
\hline
\end{tabular}

Table 3. LBC3 magnet specifications

\begin{tabular}{ll} 
Number of single pancake coils & 6 \\
Tape width [mm] & 4.03 \\
Inner radius [mm] & 7 \\
Average outer radius [mm] & 17 \\
Total number of turns [turn] & 2,658 \\
Cooling condition & Liquid helium \\
\hline
\end{tabular}

Table 4. Screening Current-Induced Field of LBC3 magnet

\begin{tabular}{cccccc}
\hline $\begin{array}{l}\text { Operating } \\
\text { current [A] }\end{array}$ & $\begin{array}{c}\text { Measured } \\
\text { field [T] }\end{array}$ & $\begin{array}{c}\text { Measured } \\
\text { SCIF [T] }\end{array}$ & $\begin{array}{c}\text { Simulated } \\
\text { field [T] }\end{array}$ & $\begin{array}{c}\text { Simulated } \\
\text { SCIF [T] }\end{array}$ & $\begin{array}{c}\text { FEM+TAM } \\
\text { SCIF [T] }\end{array}$ \\
\hline 8.96 & 0.51 & -0.013 & 0.49 & -0.035 & -0.023 \\
48.9 & 2.59 & -0.266 & 2.69 & -0.190 & -0.095 \\
\hline
\end{tabular}


Table 5. Specifications of Coil 2 of $800-\mathrm{MHz}$ REBCO insert

Total number of DP coils 32

Number of inside-notched DP coils

10

Number of turns per pancake (regular / notched)

$120 / 118$

Inner diameter of DP (regular / notched) [mm]

Outer diameter average [mm]

$150.8 / 151.4$

Overall height [mm]

169.2

Average turn-to-turn resistivity $\left[\mu \Omega \cdot \mathrm{cm}^{2}\right]$

393.9

$I_{\mathrm{c}}\left(B_{\perp}, 4.2 \mathrm{~K}\right) / I_{\mathrm{c}}(77 \mathrm{~K}$, s.f. $)$

75.4

$>2.3$ 